\title{
Group Switch-and-Stay Combining with Branch Partition for Space Diversity
}

\author{
Yawgeng A. Chau and Mostafa Al-Harbawi
}

\begin{abstract}
Group switch-and-stay combining (GSSC) with branch partitions is proposed for a low-complexity multi-antenna selection in a receiver diversity system. The GSSC can enhance the performance of conventional switch-and-stay combining (SSC), and also has a lower complexity than maximal-ratio combining (MRC) or selection combining (SC). With the GSSC scheme, multiple antenna branches are divided into non-overlapped groups. In each group the branch with the maximum signal-to-noise ratio (SNR) is used for threshold-based group switch. With particular grouping and threshold choosing, both SC and SSC schemes become different special cases of the GSSC scheme. For performance illustration, BPSK signaling on independent and identically distributed (i.i.d.) flat Rayleigh fading channels is considered, and the corresponding bit error probability (BEP) and outage probability are derived and manipulated into closed-forms. To minimize the BEP, locally optimal switch thresholds (STs) are defined and derived. For grouping with the same number of branches and an identical switch threshold (ST), the locally optimal ST becomes globally optimal. Numerical results are presented for the performance illustration and comparison.
\end{abstract}

Index Terms-Group switch-and-stay combining, space diversity, switch-and-stay combining, selection combining, optimal switch threshold.

\section{INTRODUCTION}

To combat fading effects in wireless channels, diversity combining with multiple antennas is useful for wireless communication systems [1]. Traditional combining schemes, such as MRC or SC, will use all available diversity branches, and thus have higher implementation complexity [2]. To reduce the complexity, switch-based SSC has been considered [3]-[5], where no real combiner is required. The SSC is particularly valuable for mobile stations that have limited resource and power, and it has been applied to cooperative diversity systems [6]-[8]. However, the conventional SSC scheme has a diversity order of two even if more antennas are available, and no performance improvement can be obtained by using more than two branches [9]-[11]. Moreover, the performance of SSC is much worse when compared to other combining schemes. On the other hand, although many antenna branches are available, using all of them for diversity combining is complex and expensive in implementation. Thus, the antenna selection problem is also important for many practical wireless

Manuscript received January 12, 2015; revised June 12, 2015. This work was supported by the National Science Council, Taiwan, under Grant NSC102-2220-E-155-005.

The authors are with the Department of Communications Engineering Yuan-Ze University Chung-Li, Taiwan (e-mail: eeyaw.chau @ gmail.com). communication systems [12]-[15]. In this paper, to reduce the complexity of antenna diversity and enhance reception performance of conventional SSC scheme, the GSSC is proposed and analyzed. With the GSSC, multiple branches are divided into arbitrary non-overlapped groups, and in each group, the branch with the maximum signal-to-noise ratio (SNR) is used for threshold-based group switch.

In the context, for performance evaluation, BPSK signaling on i.i.d. Rayleigh fading channels is considered, and the corresponding BEP and outage probability are derived and manipulated into closed-forms. To minimize the BEP of the GSSC scheme, the globally optimal STs will not have closed-forms and can only be obtained through numerical search, which is frequently inaccurate and intractable. Thus, to simplify the threshold design, the locally optimal ST is defined. The locally optimal ST can be derived and written in closed-form. Furthermore, if the antennas can be partitioned into groups with the same number of branches and use an identical ST, the locally optimal threshold is globally optimal.

The paper is organized as follows. In Section II, the GSSC operation is given. In Section III, the BEP and outage probability are derived In Section IV, numerical results are illustrated. Conclusions are drawn in Section V.

\section{GSSC ON RAYLEIGH FADING CHANNELS}

With GSSC, total $L$ antenna branches of a receiver diversity system are partitioned into $K$ groups $(K \geq 1)$ as shown in Fig. 1. Let $\gamma_{l}$ be the faded SNR of the signal received at the $l^{\text {th }}$ branch for $l=1,2, \cdots$, L. For i.i.d. Rayleigh fading channels, $\gamma_{l}$ is exponentially distributed with the probability density function (pdf) given by $f_{\gamma}(x)=e^{-x / \bar{\gamma}} / \bar{\gamma}$ for $x \geq 0$, where $\bar{\gamma}$ is the average SNR.

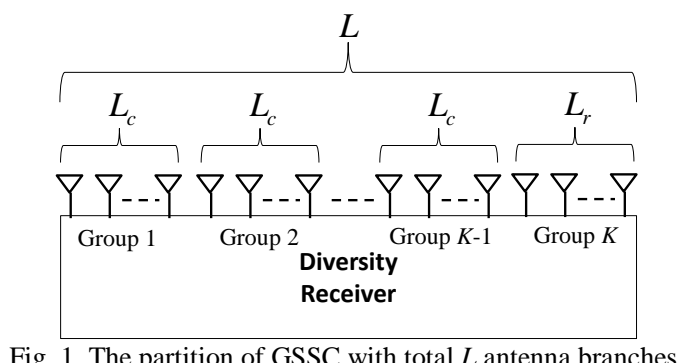

In Fig. 1, each of the first $(K-1)$ groups in the GSSC scheme contains the same number of antenna branches $L_{c}$, and the last group consists of the rest $L-(K-1) L_{c}$ antenna branches. Let $L_{r}=L-(K-1) L_{c}$. Notice that if the $L$ antennas are equally partitioned, $L_{c}=L_{r}$. 
In each group of the GSSC scheme, the branch with the maximum SNR is used for the decision of group switching. Throughout the paper, a discrete-time model is used for the switching behavior. For a reception slot at discrete-time $n$, let $\gamma_{S, k}(n)$ denote the maximum SNR for group $k$, and then $\gamma_{S, k}(n)$ is given by

$$
\gamma_{S, k}(n)=\left\{\begin{array}{lc}
\max _{1 \leq i \leq L_{c}}\left\{\gamma_{(k-1) L_{c}+i}(n)\right\}, & k=1,2, \cdots, K-1 \\
\max _{1 \leq i \leq L_{r}}\left\{\gamma_{(K-1) L_{c}+i}(n)\right\}, & k=K
\end{array} .\right.
$$

For the i.i.d. Rayleigh fading channels, the pdf of $\gamma_{S, k}$ is

$$
f_{\gamma_{S}}(x)=\left\{\begin{array}{lc}
\left(1-e^{-x / \bar{\gamma}}\right)^{L_{c}-1} \frac{L_{c} e^{-x / \bar{\gamma}}}{\bar{\gamma}}, \quad k=1,2, \cdots, K-1 \\
\left(1-e^{-x / \bar{\gamma}}\right)^{L_{r}-1} \frac{L_{r} e^{-x / \bar{\gamma}}}{\bar{\gamma}}, \quad k=K
\end{array} .\right.
$$

Let $\hat{k}(n)=k \quad(k=1,2, \cdots, K) \quad$ represent the event that branch $k$ is used for signal reception at discrete-time $n$. With GSSC, for $k=1,2, \cdots, K$, the group switching based on $\gamma_{S, k}(n)$ and the preset ST $\eta_{k}$ is characterized by

$$
\text { If } \hat{k}(n-1)=k, \hat{k}(n)=\left\{\begin{array}{cc}
k, & \text { if } \gamma_{S, k}(n) \geq \eta_{k} \\
k+1, & \text { if } \gamma_{S, k}(n)<\eta_{k} \text { and } k \leq K-1 \\
1, & \text { if } \gamma_{S, K}(n)<\eta_{k} \text { and } k=K
\end{array}\right.
$$

The above switch operation will be completed within the guard time between consecutive reception slots. If group $k$ is chosen for $k=1,2, \cdots, K$, then the branch with $\gamma_{S, k}$ is employed for the corresponding signal reception.

Notice that if $L_{c}=1$ is set, the GSSC reduces to the conventional SSC. In addition, if $L_{c}=L$ (i.e., $K=1$ without partition), the only choice of the ST is $\eta=0$, and then the GSSC becomes the traditional SC. Thus, both SSC and SC schemes are different special cases of the GSSC scheme. Throughout the following analysis, the case of $K \geq 2$ is considered.

\section{PERFormance Evaluation}

\section{A. Markov Chain Model}

For performance analysis, the Markov chain model similar to those used in [5] and [9] is applied to the group switching. In the following analysis, the time index $n$ will be omitted for notation simplification. We also assume that the event $\hat{k}=k$ is a stationary Markov chain. Then, the corresponding state transition probability $p^{i, j}$ from $\hat{k}=i$ to $\hat{k}=j$ is given by

$$
p^{i, j}=\left\{\begin{array}{cc}
\operatorname{Pr}\left(\gamma_{S, i} \geq \eta\right), & j=i ; i=1,2, \cdots, K \\
\operatorname{Pr}\left(\gamma_{S, i}<\eta\right), & j=i+1 ; i=1,2, \cdots, K-1 \\
\operatorname{Pr}\left(\gamma_{S, K}<\eta\right), & i=K, j=1 \\
0, & \text { otherwise }
\end{array} .\right.
$$

Then, it is straightforward to show that the stationary distribution of state $k$ of the Markov chain is given by

$$
\begin{aligned}
\pi_{k} & =\left(\sum_{j=1}^{K} \frac{1}{P\left(\gamma_{s, j}<\eta_{j}\right)}\right)^{-1} \frac{1}{P\left(\gamma_{s, k}<\eta_{k}\right)}, \quad k=1,2, \cdots, K \\
& =\left\{\begin{array}{cc}
\left(\sum_{j=1}^{K-1} \frac{\left(1-e^{-\eta_{k} / \bar{\gamma}}\right)^{L_{c}}}{\left(1-e^{-\eta_{j} / \bar{\gamma}}\right)^{L_{c}}}+\frac{\left(1-e^{-\eta_{k} / \bar{\gamma}}\right)^{L_{c}}}{\left(1-e^{-\eta_{K} / \bar{\gamma}}\right)^{L_{r}}}\right)^{-1}, & k=1,2, \cdots, K-1 \\
\left(\sum_{j=1}^{K-1} \frac{\left(1-e^{-\eta_{K} / \bar{\gamma}}\right)^{L_{r}}}{\left(1-e^{-\eta_{j} / \bar{\gamma}}\right)^{L_{c}}}+1\right)^{-1}, & k=K
\end{array}\right.
\end{aligned}
$$

where

$$
P\left(\gamma_{s, j}<\eta_{j}\right)=\left\{\begin{array}{lc}
\left(1-e^{-x / \bar{\gamma}}\right)^{L_{c}}, & j=1,2, \cdots, K-1 \\
\left(1-e^{-x / \bar{\gamma}}\right)^{L_{r}}, & j=K
\end{array} .\right.
$$

Notice that if an identical ST $\eta$ is employed for all diversity branches, the stationary distribution becomes

$$
\pi_{k}=\left\{\begin{array}{cc}
\frac{\left(1-e^{-\eta / \bar{\gamma}}\right)^{L_{r}}}{\left(1-e^{-\eta / \bar{\gamma}}\right)^{L_{c}}+(K-1)\left(1-e^{-\eta / \bar{\gamma}}\right)^{L_{r}}}, & k=1,2, \cdots, K-1 \\
\frac{\left(1-e^{-\eta / \bar{\gamma}}\right)^{L_{c}}}{\left(1-e^{-\eta / \bar{\gamma}}\right)^{L_{c}}+(K-1)\left(1-e^{-\eta / \bar{\gamma}}\right)^{L_{r}}}, & k=K
\end{array} .\right.
$$

\section{B. BEP Analysis}

With the above stationary distribution, the total BEP is given by

$$
P_{e}\left(\eta_{1}, \eta_{2}, \cdots, \eta_{K}\right)=\sum_{i=1}^{K} \pi_{i} \cdot P_{e \mid i}\left(\eta_{i}\right)
$$

where $P_{e \mid i}$ is the BEP conditioned on that the $i^{\text {th }}$ group is used in the previous slot for signal reception. For i.i.d. fading channels, this conditional BEP has the form

$P_{e \mid i}\left(\eta_{i}\right)=\left\{\begin{array}{c}P_{0}\left(r_{s, 1}>0, \gamma_{s, 1} \geq \eta_{i}\right)+P_{0}\left(r_{s, 1}>0\right) P_{0}\left(\gamma_{s, 1}<\eta_{i}\right) \\ \text { for } 1 \leq i \leq K-2 \\ P_{0}\left(r_{s, 1}>0, \gamma_{s, 1} \geq \eta_{K-1}\right)+P_{0}\left(r_{s, K}>0\right) P_{0}\left(\gamma_{s, 1}<\eta_{K-1}\right) \\ \text { for } i=K-1 \\ P_{0}\left(r_{s, K}>0, \gamma_{s, K} \geq \eta_{K}\right)+P_{0}\left(r_{s, 1}>0\right) P_{0}\left(\gamma_{s, K}<\eta_{K}\right) \\ \text { for } i=K\end{array}\right.$

If $K>2$, and

$P_{e \mid i}\left(\eta_{i}\right)=\left\{\begin{array}{c}P_{0}\left(r_{s, 1}>0, \gamma_{s, 1} \geq \eta_{K-1}\right)+P_{0}\left(r_{s, 2}>0\right) P_{0}\left(\gamma_{s, 1}<\eta_{K-1}\right) \\ \text { for } i=1 \\ P_{0}\left(r_{s, 2}>0, \gamma_{s, 2} \geq \eta_{K}\right)+P_{0}\left(r_{s, 1}>0\right) P_{0}\left(\gamma_{s, 2}<\eta_{K}\right) \\ \text { for } i=2\end{array}\right.$

If $K=2$, where $P_{0}(\cdot)$ denotes the error probability of 
detection when " 0 " has been transmitted by using BPSK signaling. With some manipulations for the conditional error probability given in (9), we obtain following closed-forms for different cases

$$
\begin{aligned}
& P_{e l i}\left(\eta_{i}\right)=\sum_{j=0}^{L_{c}-1} \frac{(-1)^{j}}{2}\left(\begin{array}{c}
L_{c} \\
j+1
\end{array}\right)\left[e^{-(j+1) \eta_{i} / \bar{\gamma}} \operatorname{erfc}\left(\sqrt{\eta_{i}}\right)\right. \\
&-\sqrt{\bar{\gamma} /(\bar{\gamma}+j+1)} \operatorname{erfc}\left(\sqrt{\eta_{i}(\bar{\gamma}+j+1) / \bar{\gamma}}\right) \\
&\left.+\left(1-e^{-\eta_{i} / \bar{\gamma}}\right)^{L_{c}}(1-\sqrt{\bar{\gamma} /(\bar{\gamma}+j+1)})\right]
\end{aligned}
$$

for $1 \leq i \leq K-2$,

$$
\begin{aligned}
P_{e \mid K-1}\left(\eta_{K-1}\right)= & \sum_{j=0}^{L_{c}-1} \frac{(-1)^{j}}{2}\left(\begin{array}{c}
L_{c} \\
j+1
\end{array}\right)\left[e^{-(j+1) \eta_{K-1} / \bar{\gamma}} \operatorname{erfc}\left(\sqrt{\eta_{K-1}}\right)\right. \\
& \left.-\sqrt{\bar{\gamma} /(\bar{\gamma}+j+1)} \operatorname{erfc}\left(\sqrt{\eta_{K-1}(\bar{\gamma}+j+1) / \bar{\gamma}}\right)\right] \\
+ & \left(1-e^{-\eta_{K-1} / \bar{\gamma}}\right)^{L_{c}} \sum_{j=0}^{L_{r}-1} \frac{(-1)^{j}}{2}\left(\begin{array}{c}
L_{r} \\
j+1
\end{array}\right)(1-\sqrt{\bar{\gamma} /(\bar{\gamma}+j+1)}),
\end{aligned}
$$

and

$$
\begin{aligned}
P_{e \mid K}\left(\eta_{K}\right)= & \sum_{j=0}^{L_{r}-1} \frac{(-1)^{j}}{2}\left(\begin{array}{c}
L_{r} \\
j+1
\end{array}\right)\left[e^{-(j+1) \eta_{K} / \bar{\gamma}} \operatorname{erfc}\left(\sqrt{\eta_{K}}\right)\right. \\
& \left.-\sqrt{\bar{\gamma} /(\bar{\gamma}+j+1)} \operatorname{erfc}\left(\sqrt{\eta_{K}(\bar{\gamma}+j+1) / \bar{\gamma}}\right)\right] \\
+ & \left(1-e^{-\eta_{K} / \bar{\gamma}}\right)^{L_{r}} \sum_{j=0}^{L_{c}-1} \frac{(-1)^{j}}{2}\left(\begin{array}{c}
L_{c} \\
j+1
\end{array}\right)(1-\sqrt{\bar{\gamma} /(\bar{\gamma}+j+1)}),
\end{aligned}
$$

where $\operatorname{erfc}(\cdot)$ is the complementary error function, and

$$
\left(\begin{array}{c}
M \\
j+1
\end{array}\right)=\frac{M !}{(j+1) !(M-j-1) !}, M=L_{c} \text { or } L_{r} .
$$

For the case of $K=2$, conditional error probabilities similar to (12) and (13) can be employed.

If an identical ST is used for all branches, i.e., $\eta_{k}=\eta$ for $k=1,2, \cdots, K$, the BEP reduces to the form

$$
\begin{aligned}
P_{e}(\eta) & =\frac{\left(1-e^{-\eta / \bar{\gamma}}\right)^{L_{r}}\left[(K-2) P_{e \mid 1}(\eta)+P_{e \mid K-1}(\eta)\right]}{\left(1-e^{-\eta / \bar{\gamma}}\right)^{L_{c}}+(K-1)\left(1-e^{-\eta / \bar{\gamma}}\right)^{L_{r}}} \\
& +\frac{\left(1-e^{-\eta / \bar{\gamma}}\right)^{L_{c}} P_{e \mid K}(\eta)}{\left(1-e^{-\eta / \bar{\gamma}}\right)^{L_{c}}+(K-1)\left(1-e^{-\eta / \bar{\gamma}}\right)^{L_{r}}} .
\end{aligned}
$$

\section{Optimization of $S T$}

For $K \geq 2$, to obtain the multiple optimal STs $\left(\eta_{1}, \eta_{2}, \cdots, \eta_{K}\right)$ that minimize the BEP given by (8) is difficult and intractable. On the other hand, we may define the locally optimal ST $\eta_{i}$ that minimize $P_{e \mid i}\left(\eta_{i}\right)$.

By taking the derivative of $P_{e \mid i}\left(\eta_{i}\right)$ given by (11)-(13), respectively, and solving the relevant minimization problems, we obtain the locally optimal ST $\eta_{i}$ as

$$
\eta_{i}^{*}=\left\{\begin{array}{l}
\left(\operatorname{erfc}^{-1}\left[\sum_{j=0}^{L_{c}-1}(-1)^{j}\left(\begin{array}{c}
L_{c} \\
j+1
\end{array}\right)\left(1-\sqrt{\frac{\bar{\gamma}}{\bar{\gamma}+j+1}}\right)\right]\right)^{2}, 1 \leq i \leq K-2 \\
\left(\operatorname{erfc}^{-1}\left[\sum_{j=0}^{L_{r}-1}(-1)^{j}\left(\begin{array}{c}
L_{r} \\
j+1
\end{array}\right)\left(1-\sqrt{\frac{\bar{\gamma}}{\bar{\gamma}+j+1}}\right)\right]\right)^{2}, i=K-1 \\
\left(\operatorname{erfc}^{-1}\left[\sum_{j=0}^{L_{c}-1}(-1)^{j}\left(\begin{array}{c}
L_{c} \\
j+1
\end{array}\right)\left(1-\sqrt{\frac{\bar{\gamma}}{\bar{\gamma}+j+1}}\right)\right]\right)^{2}, i=K
\end{array}\right.
$$

for $K>2$, and

$$
\eta_{i}^{*}=\left\{\begin{array}{l}
\left(\operatorname{erfc}^{-1}\left[\sum_{j=0}^{L_{r}-1}(-1)^{j}\left(\begin{array}{c}
L_{r} \\
j+1
\end{array}\right)\left(1-\sqrt{\frac{\bar{\gamma}}{\bar{\gamma}+j+1}}\right)\right]\right)^{2}, i=1 \\
\left(\operatorname{erfc}^{-1}\left[\sum_{j=0}^{L_{c}-1}(-1)^{j}\left(\begin{array}{c}
L_{c} \\
j+1
\end{array}\right)\left(1-\sqrt{\frac{\bar{\gamma}}{\bar{\gamma}+j+1}}\right)\right]\right)^{2}, i=2
\end{array}\right.
$$

for $K=2$, where $\operatorname{erfc}^{-1}(\cdot)$ is the inverse function of the complementary error function. Notice that, if $L_{c}=L_{r}$ (i.e., for equally partitioned branches) with an identical ST, the above locally optimal ST becomes globally optimal.

\section{Outage Probability}

The outage probability is given by

$$
P_{\text {out }}\left(\eta_{1}, \eta_{2}, \cdots, \eta_{K}\right)=\sum_{i=1}^{K} \pi_{i} \cdot P_{\text {out } i}\left(\eta_{i}\right)
$$

where $P_{\text {out } i}$ is the outage probability when the $i^{\text {th }}$ group is used in the previous slot of signal reception. With the outage threshold $x, P_{\text {out } i}$ for i.i.d. channels is given by

$P_{\text {out } i}=\left\{\begin{array}{l}\operatorname{Pr}\left(\gamma_{s, 1} \leq x, \gamma_{s, 1} \geq \eta_{i}\right)+\operatorname{Pr}\left(\gamma_{s, 2} \leq x, \gamma_{s, 1}<\eta_{i}\right), 1 \leq i \leq K-2 \\ \operatorname{Pr}\left(\gamma_{s, 1} \leq x, \gamma_{s, 1} \geq \eta_{K-1}\right)+\operatorname{Pr}\left(\gamma_{s, K} \leq x, \gamma_{s, 1}<\eta_{K-1}\right), i=K-1 \\ \operatorname{Pr}\left(\gamma_{s, K} \leq x, \gamma_{s, K} \geq \eta_{K}\right)+\operatorname{Pr}\left(\gamma_{s, 1} \leq x, \gamma_{s, K}<\eta_{K}\right), i=K\end{array}\right.$

for $K>2$, where the ST symbols are omitted to alleviate notations. With (19), $P_{\text {out }}$ can be obtained as

$$
P_{\text {out } i}=\left\{\begin{array}{c}
\left(1-e^{-x / \bar{\gamma}}\right)^{L_{c}}\left(1-e^{-\eta_{i} / \bar{\gamma}}\right)^{L_{c}}, \text { for } x \leq \eta_{i} ; 1 \leq i \leq K-2 \\
\left(1-e^{-x / \bar{\gamma}}\right)^{L_{c}}-\left(1-e^{-\eta_{i} / \bar{\gamma}}\right)^{L_{c}}+\left(1-e^{-x / \bar{\gamma}}\right)^{L_{c}}\left(1-e^{-\eta_{i} / \bar{\gamma}}\right)^{L_{c}}, \\
\text { for } x>\eta_{i} ; 1 \leq i \leq K-2 \\
\left(1-e^{-x / \bar{\gamma}}\right)^{L_{r}}\left(1-e^{-\eta_{K-1} / \bar{\gamma}}\right)^{L_{c}}, \text { for } x \leq \eta_{K-1} ; i=K-1 \\
\left(1-e^{-x / \bar{\gamma}}\right)^{L_{c}}-\left(1-e^{-\eta_{K-1} / \bar{\gamma}}\right)^{L_{c}}+\left(1-e^{-x / \bar{\gamma}}\right)^{L_{r}}\left(1-e^{-\eta_{K-1} / \bar{\gamma}}\right)^{L_{c}}, \\
\text { for } x>\eta_{K-1} ; i=K-1 \\
\left(1-e^{-x / \bar{\gamma}}\right)^{L_{c}}\left(1-e^{-\eta_{K} / \bar{\gamma}}\right)^{L_{r}}, \text { for } x \leq \eta_{K} ; i=K \\
\left(1-e^{-x / \bar{\gamma}}\right)^{L_{r}}-\left(1-e^{-\eta_{K} / \bar{\gamma}}\right)^{L_{r}}+\left(1-e^{-x / \bar{\gamma}}\right)^{L_{c}}\left(1-e^{-\eta_{K} / \bar{\gamma}}\right)^{L_{r}}, \\
\text { for } x>\eta_{K} ; i=K
\end{array}\right.
$$

for $K>2$. When $K=2$, we can obtain 


$$
P_{\text {out } \mid i}=\left\{\begin{array}{c}
\left(1-e^{-x / \bar{\gamma}}\right)^{L_{r}}\left(1-e^{-\eta_{1} / \bar{\gamma}}\right)^{L_{c}}, \text { for } x \leq \eta_{1} ; i=1 \\
\left(1-e^{-x / \bar{\gamma}}\right)^{L_{c}}-\left(1-e^{-\eta_{1} / \bar{\gamma}}\right)^{L_{c}}+\left(1-e^{-x / \bar{\gamma}}\right)^{L_{r}}\left(1-e^{-\eta_{1} / \bar{\gamma}}\right)^{L_{c}}, \\
\text { for } x>\eta_{1} ; i=1 \\
\left(1-e^{-x / \bar{\gamma}}\right)^{L_{c}}\left(1-e^{-\eta_{2} / \bar{\gamma}}\right)^{L_{r}}, \text { for } x \leq \eta_{2} ; i=2 \\
\left(1-e^{-x / \bar{\gamma}}\right)^{L_{r}}-\left(1-e^{-\eta_{2} / \bar{\gamma}}\right)^{L_{r}}+\left(1-e^{-x / \bar{\gamma}}\right)^{L_{c}}\left(1-e^{-\eta_{2} / / \bar{\gamma}}\right)^{L_{r}}, \\
\text { for } x>\eta_{2} ; i=2
\end{array} .\right.
$$

To evaluate the outage probability, the locally optimal STs given by (16) and (17) will be also applicable.

\section{NUMERICAL RESULTS}

The BEP and outage probability with the locally optimal STs are evaluated numerically for different cases.

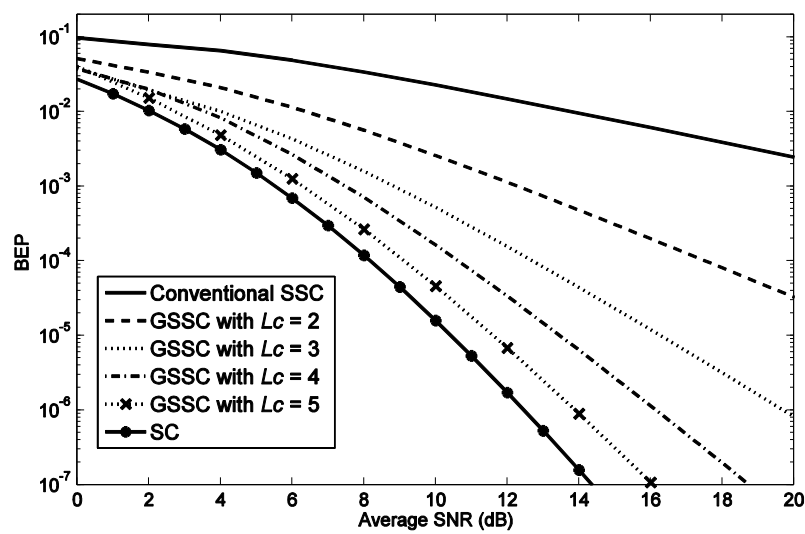

Fig. 2. The BEP comparison for $L=6$ with different partitions.

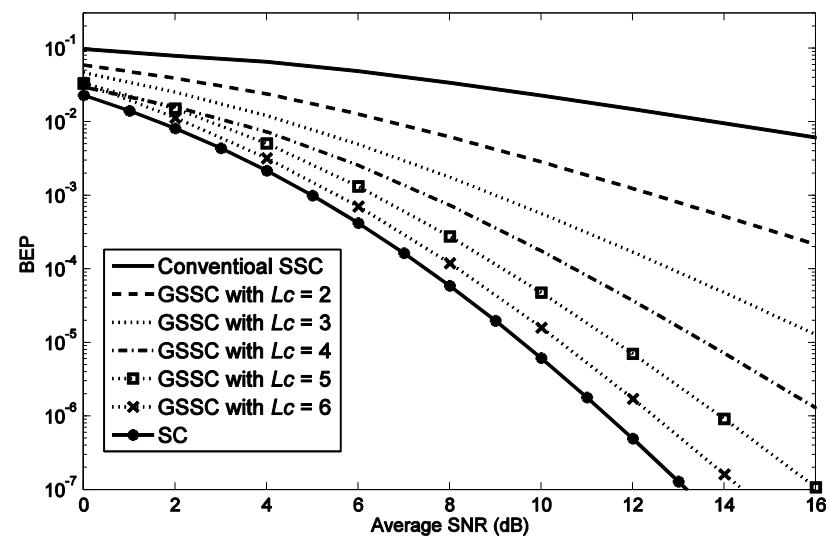

Fig. 3. The BEP comparison for $L=7$ with different partitions.

As examples for performance illustrations, the BEP with different partitions (i.e., different values of $L_{c}$ ) is illustrated for $L=6$ in Fig. 2 and for $L=7$ in Fig. 3, where the conventional SSC and SC are also plotted for comparisons. From Fig. 2 and Fig. 3, the diversity gain of GSSC is larger much larger than that of SSC, and the performance improvement enhances when the average SNR or $L_{c}$ increases. For example, with $L=6$ and $L_{c}=2$, the power gain of GSSC over SC will be more than 10dB.

The comparison for different values of $L$ with $L_{c}=2$ and 3 is shown in Fig. 4 (on the next page). From the comparison, we notice that, with the same $L_{c}$, the BEP improvement by increasing the total number of antennas is not much.

The outage probabilities with outage thresholds $x=0 \mathrm{~dB}$ and $5 \mathrm{~dB}$ are illustrated for $L=6$ in Fig. 5 and for $L=7$ in Fig. 6, where the outage probability of SC is also plotted for comparisons. From the results in Fig. 5 and Fig. 6, we notice that the outage improvement is also remarkable by increasing the value of $L_{c}$ for GSSC. In addition, the GSSC scheme has a much better outage probability than the SC scheme that uses the total number of branches equal to $L_{c}$.

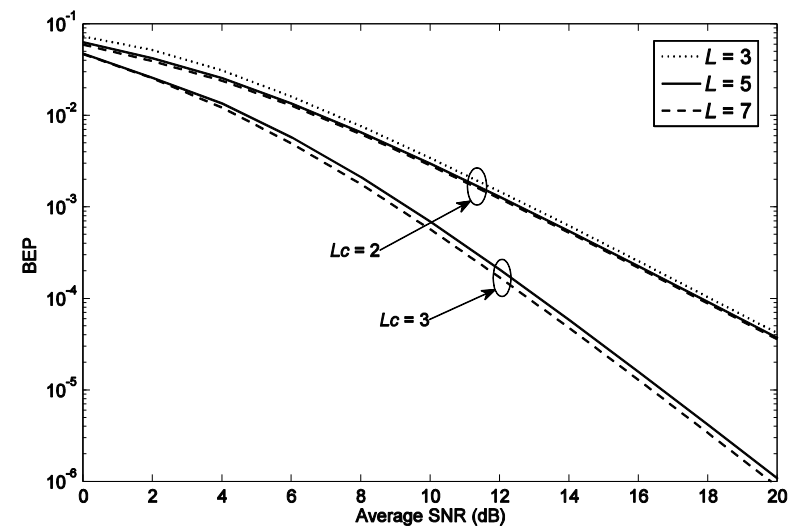

Fig. 4. The BEP comparison for different values of $\mathrm{L}$ with partitions $L_{c}=2$ and 3 .

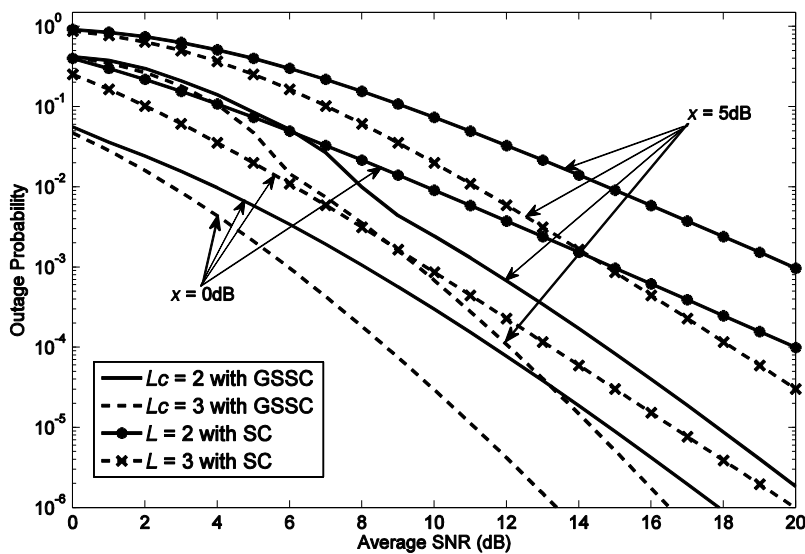

Fig. 5. The outage probability for $L=6$.

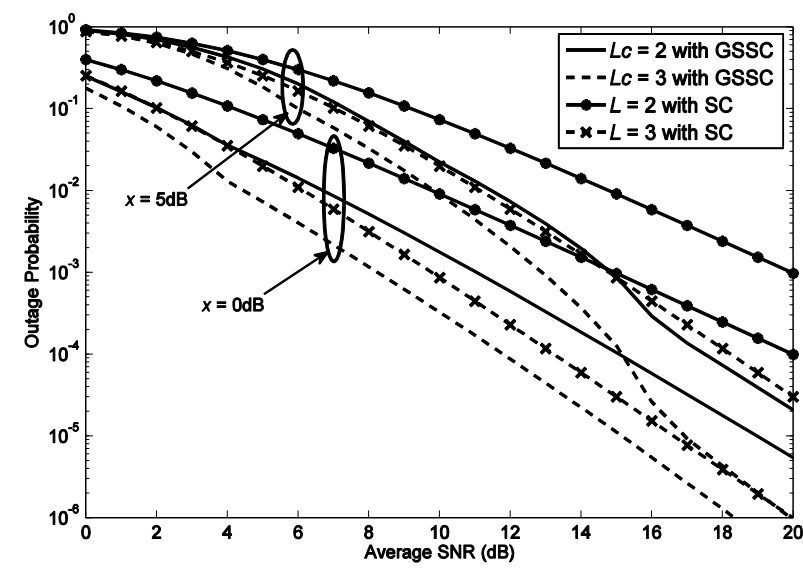

Fig. 6. The outage probability for $L=7$.

\section{CONCLUSIONS}

The GSSC scheme has been addressed, and its BEP and outage probability have been derived for performance evaluation. To minimize the BEP, locally optimal STs are 
derived. When antenna branches can be equally partitioned in some cases and an identical ST is used for all branches, the locally optimal ST also becomes globally optimal. The performance evaluation for GSSC on i.i.d. channels can be extended to independent and non-identically distributed channels. In addition, with feedback information from a receiver to a transmitter, the GSSC scheme can also be applied to transmission antenna diversity or multi-input multi-output systems.

\section{REFERENCES}

[1] M. K. Simon and M.-S. Alouini, Digital Communication over Fading Channels, John Wiley \& Sons, Inc., 2000.

[2] D. G. Brennan, "Linear diversity combining techniques," in Proc. IRE, vol. 47, pp. 1075-1102, June 1959.

[3] M. A. Blanco and K. J. Zdunek, "Performance and optimization of switched diversity systems for the detection of signals with Rayleigh fading," IEEE Trans. Commun., vol. COM-27, pp. 1887-1895, Dec. 1979.

[4] Y.-C. Ko, M.-S. Alouini, and M. K. Simon, "Analysis and optimization of switched diversity systems," IEEE Trans. Veh. Technol., vol. 49, pp. 1813-1831, Sept. 2000.

[5] C. Tellambura, A. Annamalai, and V. K. Bhargava, "Unified analysis of switched diversity systems in independent and correlated fading channel," IEEE Trans. Commun., vol. 49, pp. 1955-1965, Nov. 2001

[6] D. S. Michalopoulos and G. K. Karagiannidis, "Distributed switch and stay combining (DSSC) with a single decode and forward relay," IEEE Commu. Lett., vol. 11, pp. 408-410, May 2007.

[7] V. N. Q. Bao and H. Y. Kong, "Distributed switch and stay combining for selection relay networks," IEEE Commun. Lett., vol. 13, no. 12, pp. 914-916, Dec. 2009.

[8] M. Yan et al., "Outage probability of switch and stay combining in two-way amplify-and-forward relay networks," IEEE Wireless Comm. Lett., vol. 1, pp. 296-299, Aug. 2012.
[9] H. -C. Yang and M.-S. Alouini, "Performance analysis of multibranch switched diversity systems," IEEE Trans. Commun., vol. 51, pp. 782-794, May 2003.

[10] R. Prasad, Universal Wireless Personal Communications, Boston: Artech House, 1998.

[11] K.-H. Liu, "On the performance of time-orthogonal incremental relaying based on demodulate-and-forward with distributed channel access," IEEE Trans. Veh. Technol., vol. 61, pp.737-747, Feb. 2012.

[12] A. Gorokhov, D. Gore, and A. Paulraj, "Receive antenna selection for MIMO flat-fading channels: Theory and algorithms," IEEE Trans. Inf. Theory, pp. 2687-2696, Oct. 2003

[13] A. F. Molisch and M. Z. Win, "MIMO systems with antenna selection," IEEE Microwave Mag., vol. 5, pp. 46-56, Mar. 2004

[14] V. Kristem, N. B. Mehta, and A. F. Molisch, "Optimal receive antenna selection in time-varying fading channels with practical training Constraints," IEEE Trans. Commun., vol. 58, pp. 2023-2034, July 2010.

[15] N. B. Mehta, S. Kashyap, and A. F. Molisch, "Antenna selection in LTE: From motivation to specification," IEEE Commun. Mag., vol. 50, pp. 144-150, Oct. 2012.

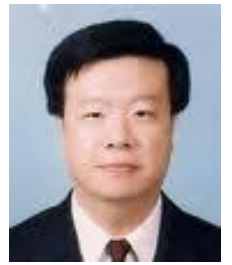

Yawgeng A. Chau received his Ph.D. degree in electrical engineering from University of Maryland-College Park, U.S.A. He is currently a professor and the chairman of the Department of Communications Engineering, Yuan-Ze University, Taiwan. His interests are in the areas of wireless communications and corresponding implementations. Gemtek Co. Ltd., Taiwan. He also serves as a consultant and board member of

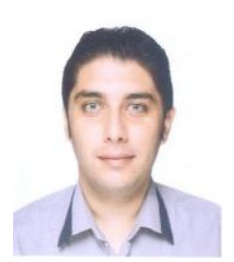

Mostafa Al-Harbawi received his Ph.D. degree in communications engineering from Yuan-Ze University, Taiwan. His interest is in wireless communications. 